Trauma Berufskrankh 2012 · 14[Suppl 3]:327-330 DOI 10.1007/s10039-011-1828-7

Online publiziert: 22. März 2012

(c) Springer-Verlag 2012

\section{U.C. Liener}

Klinik für Orthopädie, Unfallchirurgie und Wiederherstellungschirurgie,

Zentrum für Schwerbrandverletzte, Vinzenz von Paul Kliniken gGmbH, Marienhospital Stuttgart

\section{Hämatogene Spondylitis}

\section{Hätten sie es gewusst?}

\section{Kasuistik \\ Vorgeschichte}

Ein 72-jähriger Patient wurde mit akuten Rückenschmerzen vom Notarzt in die Klinik gebracht. Während des Transports musste aufgrund der Beschwerden Fentanyl verabreicht werden.

Der Patient berichtete, dass seit Jahren Schmerzen im Rücken bestünden. Am Vortag hätte er einen Koffer angehoben und seither hätten die Beschwerden an Intensität stark zugenommen. Bei der Aufnahme litt er an starken krampfartigen Rückenschmerzen, es wurden ein Klopf- und Druckschmerz der Lendenwirbelsäule (LWS) festgestellt, neurologische Ausfälle lagen nicht vor.

An Nebenerkrankungen waren ein Zustand nach Aortenklappenersatz, eine Herzinsuffizienz NYHA 2 (New York Heart Association) sowie eine chronisch obstruktive Lungenerkrankung zu verzeichnen.

Der Patient berichtete, dass 6 Monate zuvor eine Magnetresonanztomographie (MRT) der LWS angefertigt worden sei. Laut vorliegendem Fremdbefund wurden hierbei Protrusionen von L1/2 (L: lumbal) sowie L2/3 und ein mediolateraler Prolaps L5/S1 (S: sakral) sowie eine Spinalkanalstenose nachgewiesen.

\section{Aufnahme}

Labor. Das Aufnahmelabor war in Anbetracht der Begleiterkrankungen unauf- fällig: Leukozyten 12.700/ $\mu$ l, Hämatokrit 45\%, CRP (C-reaktives Protein) 40,7 mg/l.

Bildgebung. Eine MRT-Untersuchung war aufgrund des vorausgegangenen Aortenklappenersatzes mit dem im Hause vorhandenen 3-T-MRT nicht durchführbar, die LWS wurde daher mittels Computertomographie (CT) untersucht (• Abb. 1), welche multisegmentale degenerative Veränderungen L2-S1 mit einer daraus resultierenden Spinalkanalstenose sowie Protrusionen in den Segmenten L1/2 und L2/3 ergab.

Unter der Diagnose einer exazerbierten Schmerzsymptomatik bei Spinalkanalstenose und Osteochondrose wurde der Patient zur Analgesie und physiotherapeutischen Beübung stationär aufgenommen.

\section{Stationärer Verlauf}

Am Tag nach der Aufnahme klagte der Patient über persistierende Beschwerden im Bereich der Wirbelsäule sowie neu hinzugetretene krampfartige abdominale Schmerzen.

\section{Diagnostik}

Labor. Leukozyten $9900 / \mu l$, Hämatokrit 46\%, CRP $391 \mathrm{mg} / \mathrm{l}$, D-Dimere 3,75 mg/l (Normwert $<0,5$ ).
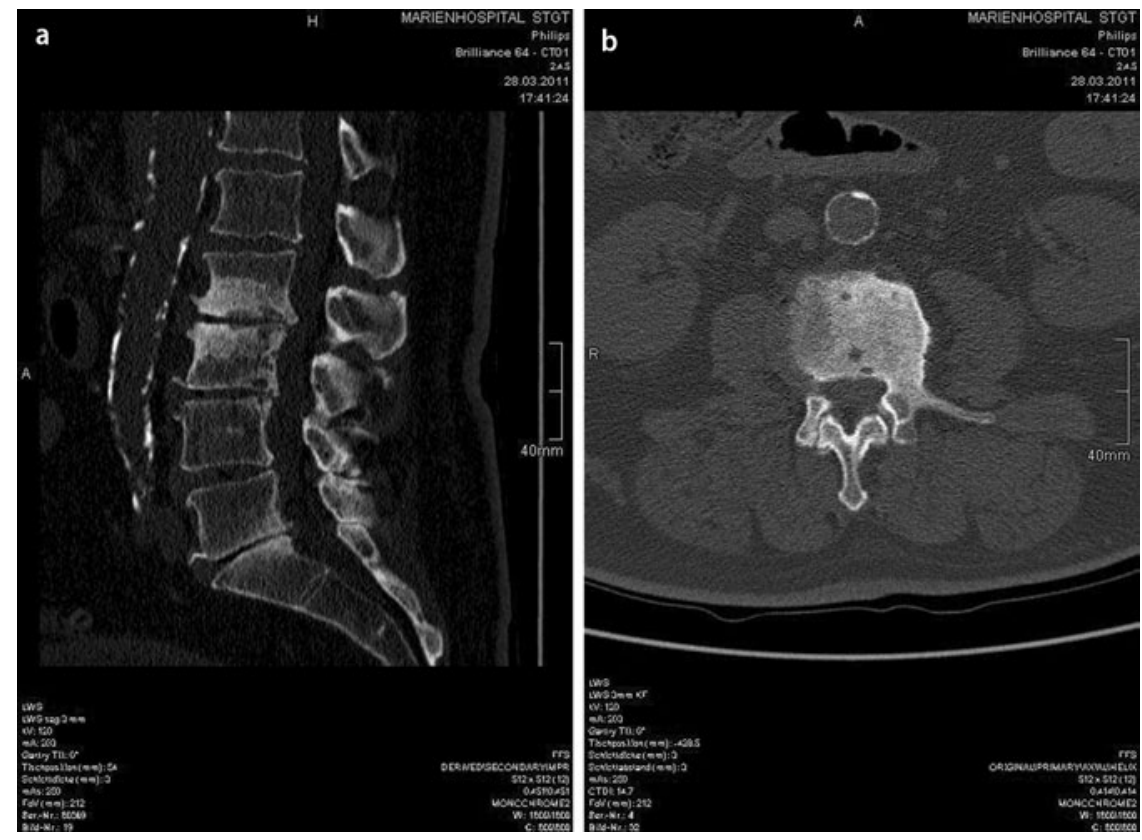

Abb. $1 \Delta$ Computertomogramm der Lendenwirbelsäule bei der Aufnahme 


\section{Hätten Sie's gewusst?}

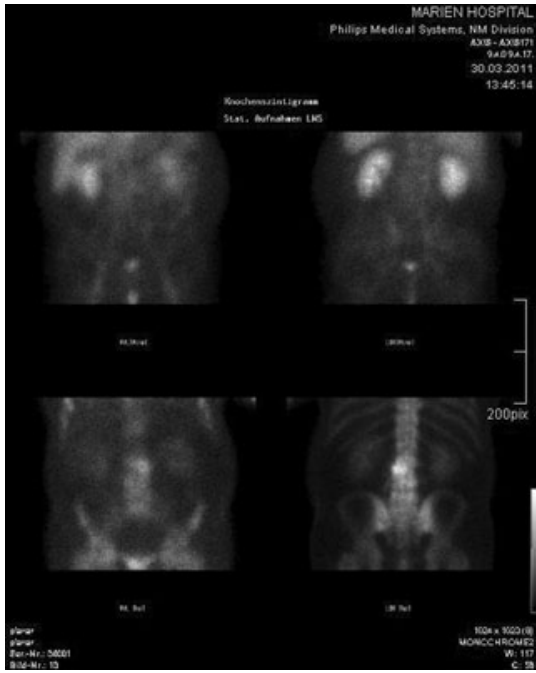

Abb. $2 \triangle$ Präoperative Skelettszintigraphie mit Mehranreicherung im Segment L2/3

Bildgebung. In der Abdomensonographie zeigte sich ein Gallenblasenhydrops. Daraufhin wurden ein CT des Abdomens sowie zum Ausschluss einer Lungenarterienembolie ein CT des Thorax durchgeführt. Diese ergaben bis auf Dystelektasen und eine Steatosis hepatis einen unauffälligen Befund.

Blutkultur. In ihr konnte Enterococcus faecalis nachgewiesen werden. Daraufhin wurde unmittelbar eine resistenzgerechte Antibiotikabehandlung mit Cefuroxim und Gentamycin begonnen.

Szintigraphie und MRT. Bei weiter unklarem Fokus wurde eine Skelettszintigraphie durchgeführt, die Hinweise auf eine Spondylitis im Bereich der Lendenwirbelsäule ergab ( $\bullet$ Abb. 2). Um das genaue Ausmaß der entzündlichen Aktivitäten und einer evtl. Myelonbeteiligung auszuschließen, erfolgte auswärtig eine 1,5-T-MRT, wobei eine Spondylitis L2/3 ohne Myelonkompression mit Affektion der paravertebralen Weichteile nachgewiesen wurde ( $\bullet$ Abb. 3).

\section{Therapie}

Bei rückläufigen Entzündungsparametern klagte der Patient weiterhin über persistierende starke Schmerzen, weswegen die dorsale transkutane Spondylodese L1L4 vorgenommen wurde. Der intraoperativ entnommene Abstrich ergab kein Keimwachstum.
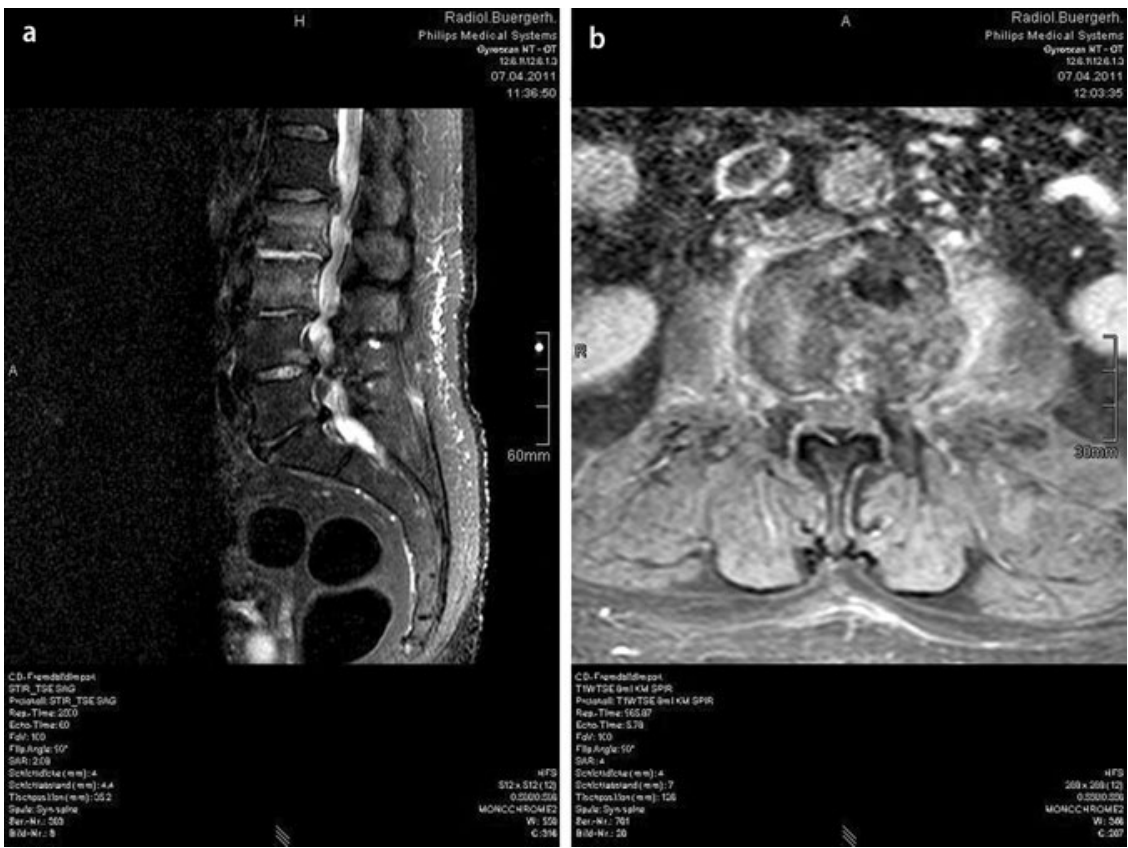

Abb. $3 \Delta$ Präoperative Magnetresonanztomographie: deutliches Ödem in den Wirbelkörpern L2 und L3 in T2-Wichtung (a) sowie in den paravertebralen Weichteilen in T1-Wichtung (b)
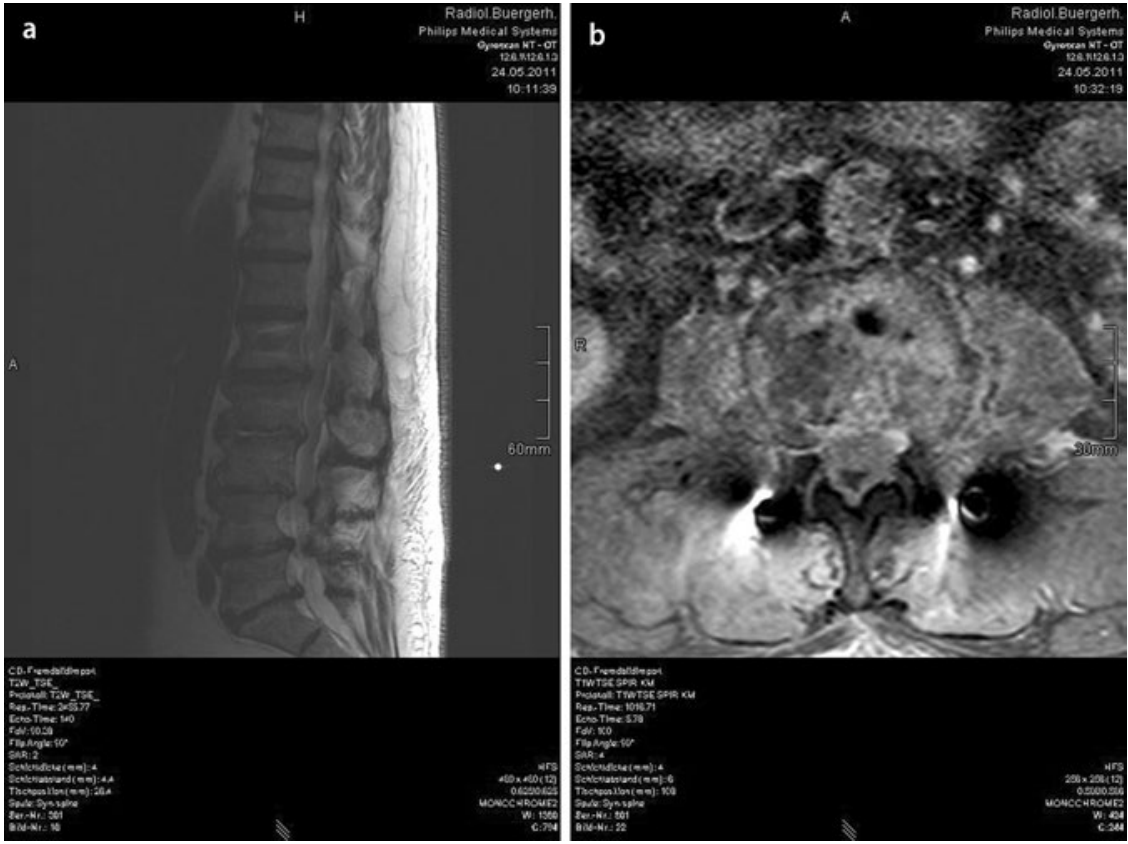

Abb. $4 \Delta$ Magnetresonanztomographie 3 Monate postoperativ, a T2-Wichtung, b T1-Wichtung mit noch geringem Restödem

Im Rahmen einer Kontroll-MRT-Untersuchung 3 Monate postoperativ zeigte sich bei dem beschwerdefreien $\mathrm{Pa}$ tienten eine Ausheilung der Spondylitis (- Abb. 4). Die Laborwerte betrugen $8700 / \mu \mathrm{l}$ und CRP $20 \mathrm{mg} / \mathrm{l}$. Daraufhin wurde die Langzeitantibiose abgesetzt.

\section{Diskussion}

Wie sich an unserem Fall exemplarisch demonstrieren lässt, stellt die Behandlung der Spondylitis bzw. Spondylodiszitis weiterhin eine Herausforderung dar. Ihre Inzidenz beträgt etwa 2,2:100.000, wobei in 
den letzten Jahren eine Zunahme zu verzeichnen war, welche auf die zunehmend ältere Bevölkerung mit der daraus resultierenden Multimorbidität sowie auf verbesserte diagnostische Möglichkeiten mit einer höheren Spezifität zurückgeführt werden kann [1]. Am häufigsten betroffen ist die lumbale Wirbelsäule [7]. Der Infektion der Wirbelsäule geht häufig eine Infektion in anderen Bereichen des Körpers voraus [6].

Die Diagnose ist in der Frühphase der Erkrankung aufgrund der unspezifischen Symptome häufig schwierig zu stellen. Dies spiegelt sich in dem Intervall zwischen dem Auftreten von Symptomen bis zur Diagnosestellung, welches in der Literatur mit bis zu 9 Monaten angegeben wird, wider [8]. Die Beschwerden sind unspezifisch, häufig besteht jedoch ein axialer Kompressionsschmerz der Wirbelsäule [7]. Die Diagnostik beinhaltet zum einen Laboruntersuchungen, in denen sich am häufigsten eine Leukozytose sowie eine CRP- bzw. BSGErhöhung (BSG: Blutsenkungsgeschwindigkeit) zeigen. Entscheidungsrelevant für die Therapie sind positive Blutkulturen. Da diese im Mittel jedoch nur in etwa $60 \%$ der Fälle positiv sind, ist zur weiteren Abklärung häufig eine interventionelle Punktion notwendig, in welcher in gleicher Sitzung eine Drainage gelegt werden kann [7]. Am häufigsten können hierbei als verursachende Erreger Staphylococcus aureus gefolgt von Escherichia coli (E. coli) und koagulasenegativen Streptokokken nachgewiesen werden [6].

Zur Differenzialdiagnose ist die MRT mit einer Genauigkeit von 90\% in der Diagnostik einer Spondylitis am besten geeignet. Typisch sind hierbei eine Signalintensität in der T2-gewichteten Sequenz sowie häufig eine Zerstörung der Grundund Deckplatten [9]. Da mittels MRT eine Spondylitis sensitiver als mittels CT nachgewiesen werden kann, besteht die Indikation zu Letzterer, wenn - wie auch im hier vorgestellten Fall - die Durchführung einer MRT kontraindiziert ist. Insbesondere in der Frühphase der Erkrankung ist es schwierig, in der CT zwischen einer Spondylitis und einer erosiven Osteochondrose zu unterscheiden [2]. Die Skelettszintigraphie liefert bereits einige Tage nach Beginn der Symptome einen

Trauma Berufskrankh 2012 · 14[Suppl 3]:327-330 DOI 10.1007/s10039-011-1828-7

(c) Springer-Verlag 2012

\section{U.C. Liener}

\section{Hämatogene Spondylitis. Hätten sie es gewusst?}

\section{Zusammenfassung}

Die hämatogene Spondylitis ist die häufigste Manifestation einer hämatogenen Osteomyelitis im Erwachsenenalter. In den letzten Jahren ist aufgrund der zunehmenden Multimorbidität der älter werdenden Bevölkerung eine steigende Inzidenz zu beobachten. Die Diagnosestellung erfolgt wegen der unspezifischen Symptome und der hohen Prävalenz von Rückenbeschwerden in der Frühphase häufig verzögert. Am häufigsten werden als Erreger Staphylococcus aureus und Escherichia coli (E. coli) isoliert, wobei die Lendenwirbelsäule am häufigsten betroffen ist. Aufgrund ihrer hohen Sensitivität eignet sich die Magnetresonanztomographie am besten zum Nachweis einer Infektion. Der Keimnachweis sollte durch Biopsie oder Blutkultur erfolgen. In der Frühphase der Erkrankung kann diese durch eine resistenzgerechte antibiotische Behandlung zur Ausheilung gebracht werden. Die Indikation zum chirurgischen Vorgehen ergibt sich aus einer bestehenden Instabilität, neurologischen Symptomen aufgrund einer Myelonkompression, einer progredienten Kyphose sowie persistierenden Beschwerden.

\section{Schlüsselwörter}

Osteomyelitis · Spondylitis · Staphylococcus aureus · Escherichia coli . Therapie

\section{Hematogenous spondylitis. Would you have known that?}

\section{Abstract}

Vertebral osteomyelitis is the main manifestation of hematogenous osteomyelitis in patients aged over 50 years. Because of an increasing multimorbidity of the primarily elderly patient population the incidence is increasing. Diagnosis is often delayed due to the rarity of the disease, the uncharacteristic symptoms and the high frequency of low back pain in the general population. Staphylococcus aureus followed by Escherichia coli (E. coli) are the most commonly isolated pathogens and the lumbar spine the most commonly affected area. Magnetic resonance imaging is the preferred radiologic modality because of its sensitivity. Comput- er tomography guided bone biopsy or blood cultures are crucial in the evaluation of the infection. Antimicrobial therapy of at least 6 weeks duration is recommended and should be tailored to the pathogens recovered from the culture. Acute vertebral osteomyelitis can usually be treated with antibiotics alone. Surgery is indicated in cases of persistent instability related pain, neurologic symptoms and abscess formation.

\section{Keywords}

Osteomyelitis · Spondylitis · Staphylococcus aureus - Escherichia coli - Treatment positiven Befund, problematisch ist ihre wesentlich geringere Genauigkeit, welche in der Literatur mit $67 \%$ angegeben wird [10]. Aufgrund der niedrigen Sensitivität der Leukozytenszintigraphie sind beide szintigraphischen Verfahren in der Primärdiagnostik heutzutage zugunsten der MRT in den Hintergrund gerückt [2].

Wann immer möglich, sollte die antibiotische Behandlung direkt und gezielt erregerspezifisch erfolgen. In der Frühphase der Erkrankung lässt sich durch eine gezielte antibiotische Behandlung in nahezu 90\% der Fälle eine Ausheilung erzielen $[6,10]$. Bezüglich deren optimaler Dauer existieren in der Literatur keine Daten aus kontrollierten Untersuchungen, die diesbezüglichen Empfehlungen sind daher sehr heterogen und reichen von 6 Wochen bis zu 3 Monaten [10, 11].

Die Indikation zur chirurgischen Vorgehensweise ergibt sich aus einer bestehenden Instabilität, neurologischen Symptomen aufgrund einer Myelonkompression, einer progredienten Kyphose sowie persistierenden Beschwerden [3] Einen Meilenstein in der Behandlung der tuberkulösen Spondylitis stellte die von Hodgson u. Stock [5]1956 publizierte anteriore Fusion mit kortikospongiösem Span nach Débridement der Läsion dar. Die kombinierte ventrodorsale Vorgehensweise ist bei Abszedierungen und Myelonkompression weiterhin als Stan- 
dardvorgehen anzusehen. Wie im hier vorgestellten Fall lassen sich jedoch in der Frühphase der Erkrankung durch eine isolierte perkutane dorsale Spondylodese eine rasche Beschwerdelinderung und ein Ausheilen der Infektion erzielen [3, 4].

\section{Korrespondenzadresse}

\section{Prof. Dr. U.C. Liener}

Klinik für Orthopädie, Unfallchirurgie und Wiederherstellungschirurgie, Zentrum für Schwerbrandverletzte, Vinzenz von Paul Kliniken gGmbH, Marienhospital Stuttgart, Böheimstraße 37, 70199 Stuttgart ulrichliener@vinzenz.de

Interessenkonflikt. Der korrespondierende Autor gibt an, dass kein Interessenkonflikt besteht.

The supplement containing this article is not sponsored by industry.

\section{Literatur}

1. Belzunegiu JL, Intxausti JJ, Dios JR et al (2009) Haematogenous vertebral osteomyelitis in the elderly. Clin Rheumatol 19:344-347

2. Champsaur P, Parlier-Cucau C, Juhan V (2000) Differential diagnosis of infective spondylodiscitis and erosive degenerative disc disease. J Radiol 81:516-522

3. Chen WH, Jiang LS, Dai LY (2007) Surgical treatment of pyogenic vertebral osteomyelitis with spinal instrumentation. Eur Spine J 6:1307-1316

4. Deininger $\mathrm{MH}$, Unfried MI, Voigioukas VI, Hubbe U (2009) Minimally invasive dorsal percutaneous spondylodesis for the treatment of adult pyogenic spondylodiscitis. Acta Neurochir 151:1451-1457

5. Hogdson AR, Stock FE (1956) Anterior spinal fusion. A preliminary communication on the radical treatment of Pott's disease and Pott's paraplegia. Br J Surg 44:266-275

6. McHenry MC, Easley KA, Locker GA (2002) Vertebral oosteomyelitis: long term outcome for 253 patients from 7 Cleveland area hospitals. Clin Infect Dis 34:1342-1350

7. Mylona E, Samarkos M, Kakalou E et al (220) Pyogenic vertebral osteomyelitis: a systematic review of clinical characteristics. Semin Arthritis Rheum 39:10-17

8. Nolla JM, Ariza J, Gomez-Vaquero C (2002) Spontaneous pyogenic vertebral osteomyelitis in non drug users. Semin Arthritis Rheum 31:271-278

9. Palestro CJ, Love C, Miller TT (2006) Infections and musculoskeletal conditions: imaging of musculoskeletal infections. Best Pract Res Clin Rheumatol 20:1197-1218

10. Roblot F, Besnier JM, Juhel L (2007) Optimal duration of antibiotic therapy in vertebral osteomyelitis. Semin Arthritis Rheum 36:269-277

11. Zimmerli W (2010) Vertebral osteomyelitis. N Engl J Med 362:1022-1029 